\title{
Complicaciones en embarazos logrados por reproducción asistida
}

José M. Madrazo-Cabo, Grecia A. León-Durán, Adriana Oliveros-Montiel, José A. Ledesma-Montes, Iván D. Morales-González y Virginia Sedeño-Monge*

Universidad Popular Autónoma del Estado de Puebla, Decanato de Ciencias de la Salud, Facultad de Medicina, Puebla, México

\section{Resumen}

Introducción: Se ha documentado que los embarazos por técnicas de reproducción asistida (TRA) presentan mayor riesgo de efectos adversos. Objetivo: Proporcionar evidencia de las complicaciones obstétricas y perinatales asociadas a concepciones mediante TRA versus embarazos espontáneos. Método: Revisión de artículos originales publicados entre 2010 y 2018, que abordan complicaciones obstétricas y perinatales de mayor frecuencia en embarazos por fertilización in vitro (FIV) e inyección intracitoplasmática de espermatozoides (ICSI) comparados con concepciones espontáneas. Resultados: Se incluyeron 37 artículos originales, 26 de cohortes y 11 de casos y controles. Las concepciones por FIV e ICSI se asociaron con más complicaciones obstétricas y perinatales como bajo peso al nacimiento, prematuridad, menor peso para la edad gestacional, ingreso a la unidad de cuidados intensivos neonatales, malformaciones congénitas, cesárea, ruptura prematura de membranas, entre otras. Conclusiones: Las concepciones por TRA se asocian con mayor riesgo de complicaciones obstétricas y perinatales en comparación con las espontáneas. Es necesario realizar estudios adicionales que determinen qué aspectos derivan en mayor riesgo.

PALABRAS CLAVE: Reproducción humana asistida. Fertilización in vitro. Inyección intracitoplasmática de espermatozoides.

\section{Complications in pregnancies achieved by assisted reproduction}

\begin{abstract}
Introduction: Pregnancies resulting from assisted reproductive technologies (ART) have been documented to have a higher risk of adverse effects. Objective: To provide evidence on obstetric and perinatal complications associated with conceptions by ART versus spontaneous pregnancies. Method: Comprehensive review of original articles published between 2010 and 2018 addressing the most common obstetric and perinatal complications in pregnancies resulting from in vitro fertilization (IVF) and intracytoplasmic sperm injection (ICSI) in comparison with spontaneous conceptions. Results: Thirty-seven original articles, which reported on 26 cohort studies and 11 case-control trials, were included. IVF and ICSI conceptions were associated with a larger number of obstetric and perinatal complications such as low birth weight, prematurity, low weight for gestational age, admission to the neonatal intensive care unit, congenital malformations, $C$-section and premature rupture of membranes, among others. Conclusions: Pregnancies by ART are associated with an increased risk of obstetric and perinatal complications in comparison with spontaneous conceptions. Further research is needed to determine which aspects result in higher risk.
\end{abstract}

KEY WORDS: Assisted human reproduction. In vitro fertilization. Intracytoplasmic sperm injection. 


\section{Introducción}

Más de 200000 bebés nacen en todo el mundo cada año por técnicas de reproducción asistida (TRA); ${ }^{1,2}$ a la fecha, aproximadamente han nacido 7 millones de niños concebidos mediante estos procedimientos.

En la fertilización in vitro (FIV), los ovarios son estimulados con medicamentos y posteriormente son aspirados de los folículos ováricos y fecundados in vitro; los ovocitos recuperados se mezclan con los espermatozoides en un medio de cultivo, para después ser transferidos a la cavidad uterina. ${ }^{3}$

Como parte de un ciclo de FIV se inyecta un único espermatozoide en el citoplasma de un ovocito maduro (inyección intracitoplasmática de espermatozoides, ICSI), método eficaz para ayudar a la fertilización en hombres con parámetros subóptimos del semen o con tasas de fertilización bajas.

A medida que se acumuló experiencia, las tasas de éxito aumentaron y se ampliaron las indicaciones para estos procedimientos con poca evaluación formal de sus efectos sobre el bienestar materno y fetal. ${ }^{4}$ Diversos estudios han señalado que las TRA pueden generar mayor riesgo de complicaciones maternas y perinatales en comparación con las concepciones espontáneas. ${ }^{5}$

\section{Método}

Se realizó una revisión de artículos originales publicados en revistas indexadas entre 2010 y 2018 en PubMed, Medline, CONRICyT, Medigraphic y SciELO. Las palabras clave utilizadas fueron "assisted reproductive techniques", "in vitro fertilization", "FIV", "intracytoplasmic sperm injection", "ICSI", "perinatal outcomes" y "obstetric outcomes". Se incluyeron artículos que compararan complicaciones obstétricas y perinatales entre embarazos por FIV e ICSI y concepciones espontáneas, tanto estudios de cohortes como de casos y controles. Se excluyeron metaanálisis, revisiones sistemáticas y artículos que no presentaran a los embarazos espontáneos como grupo control.

\section{Resultados}

Se incluyeron 37 estudios, 26 de cohortes y 11 de casos y controles. Los datos de cada uno se muestran en la Tabla 1.
Los resultados se describen conforme el tipo de complicación y en qué tipo de artículos el riesgo relativo de encontrar estas complicaciones fue estadísticamente mayor $(p<0.05)$ en embarazos por TRA comparados con concepciones espontáneas.

\section{Complicaciones}

- Prematuridad: complicación reportada en 26 artículos (70\% del total), en 67186 embarazos por FIV-ICSI; la edad gestacional al nacimiento registrada osciló entre las 32 y 37 semanas. En 18 artículos, la frecuencia de prematuridad fue significativamente mayor con TRA en comparación con los embarazos espontáneos. ${ }^{6-23}$

- Bajo peso al nacimiento: en 21 artículos (59 140 nacimientos por TRA) se reportó esta complicación (57\% del total). Se consideró bajo peso al nacimiento menos de $2500 \mathrm{~g}$ y muy bajo peso menos de $1500 \mathrm{~g}$; en cinco artículos se reportaron valores significativos para muy bajo peso ${ }^{6,12,17,18,22}$ y en 14 para bajo peso en los embarazos por TRA, comparados con los embara-

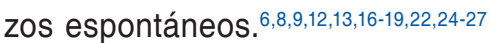

- Mortalidad perinatal: esta complicación es definida como la muerte intrauterina de un feto con peso menor de $500 \mathrm{~g}$ o desarrollo gestacional menor de 20 semanas y la de neonatos dentro de los primeros siete días de vida. 9,18,28,29 De 18 artículos (49\% del total) que incluían 591 embarazos por FIV-ICSI y en los que se reportó mortalidad perinatal, siete registraron valores significativos de mortalidad en los embarazos mediante TRA en comparación con los espontáneos. ${ }^{12,17,18,22,23,30,31}$

- Pequeño para edad gestacional (PEG): se define como tal al niño con peso al nacimiento inferior al percentil $75^{28}$ o peso menor a 2 desviaciones estándar de la población para esa edad gestacional. ${ }^{17}$ En 13 artículos (35\% del total) que incluían 10631 FIV-ICSI se reportó esta complicación; en cuatro el resultado fue significativo. . $2,17,24,32^{2}$

- Ingreso a la unidad de cuidados intensivos neonatales (UCIN): esta complicación fue reportada en ocho artículos que incluía 604 FIV-ICSI; cinco estudios mostraron diferencia significativa en los embarazos obtenidos mediante TRA. ${ }^{9,18,19,22,23}$

- Malformaciones congénitas: Veinte artículos, $54 \%$ de total que incluyeron 7288 FIV-ICSI, mencionan malformaciones congénitas; las cardiovasculares fueron las más frecuentes en 10 artículos. En comparación con concepciones 
Tabla 1. Características de estudios publicados en revistas indexadas entre 2010 y 2018, sobre complicaciones obstétricas y perinatales en embarazos por FIV e ICSI y concepciones espontáneas

\begin{tabular}{|c|c|c|c|c|c|}
\hline Estudio & País, periodo & Diseño & Población & Complicaciones reportadas & \\
\hline $\begin{array}{l}\text { Bassioun et al., } \\
2014^{23}\end{array}$ & $\begin{array}{l}\text { Egipto, } \\
\text { enero } \\
2010 \text {-diciembre } \\
2012\end{array}$ & $\begin{array}{l}\text { Casos y } \\
\text { controles }\end{array}$ & $\begin{array}{l}\text { Casos: } 739 \text { ICSI } \\
\text { Controles: } 843 \text { embarazos } \\
\text { espontáneos }\end{array}$ & $\begin{array}{l}\text { El grupo de ICSI se asoció con mayor incidencia de } \\
\text { gemelos } 12.7 \%(p<0.001) \text {, parto prematuro } 3.8 \% \\
(p=0.022) \text {, RPM } 4.6 \%(p=0.001) \text {, cesárea } 74.1 \% \\
(p<0.001) \text { y muertes neonatales } 10.4 \%(p<0.001)\end{array}$ & 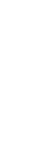 \\
\hline $\begin{array}{l}\text { Boulet et al., } \\
2016^{35}\end{array}$ & $\begin{array}{l}\text { Estados Unidos, } \\
2000-2010\end{array}$ & $\begin{array}{l}\text { Cohorte } \\
\text { retrospectiva }\end{array}$ & $\begin{array}{l}\text { Cohorte expuesta: } 64861 \\
\text { FIV-ICSI } \\
\text { Cohorte no expuesta: } \\
4553215 \text { embarazos } \\
\text { espontáneos }\end{array}$ & $\begin{array}{l}\text { La prevalencia de uno o más defectos no } \\
\text { cromosómicos fue de } 58.59 / 10000 \text { niños concebidos } \\
\text { por TRA }(n=389) \text { versus } 47.50 / 10000 \text { en niños } \\
\text { concebidos sin TRA }(n=22036) ; \text { RMa }=1.28 \\
\text { IC } 95 \%=1.15-1.42\end{array}$ & $\frac{\dot{c}}{\frac{c}{6}}$ \\
\hline $\begin{array}{l}\text { Caserta et al., } \\
2014^{9}\end{array}$ & $\begin{array}{l}\text { Italia, } \\
\text { enero 2007-junio } \\
2011\end{array}$ & $\begin{array}{l}\text { Casos y } \\
\text { controles }\end{array}$ & $\begin{array}{l}\text { Casos: } 138 \text { FIV-ICSI } \\
\text { Controles: } 207 \text { embarazos } \\
\text { espontáneos }\end{array}$ & $\begin{array}{l}\text { Edad gestacional y peso al nacimiento fueron menores } \\
\text { en el grupo con TRA. Las frecuencias de diabetes } \\
\text { y desprendimiento de placenta fueron mayores en } \\
\text { TRA en comparación con embarazos espontáneos. } \\
\text { Embarazos por TRA tuvieron mayor frecuencia de } \\
\text { desprendimiento de placenta (RM = 7.45, } \\
\text { IC } 95 \%=2.05-26.98) \text { y persistencia de conducto } \\
\text { arterioso (RM }=3.39 \text {, IC } 95 \%=1.01-11.46) \text {. }\end{array}$ & 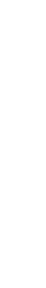 \\
\hline $\begin{array}{l}\text { Farhi et al., } \\
2013^{20}\end{array}$ & $\begin{array}{l}\text { Israel, } \\
\text { junio } 2006- \\
\text { diciembre } 2008\end{array}$ & $\begin{array}{l}\text { Cohorte } \\
\text { prospectiva }\end{array}$ & $\begin{array}{l}\text { Cohorte expuesta: } 561 \text { TRA } \\
\text { (223 FIV y } 338 \text { ICSI) } \\
\text { Cohorte no expuesta: } 600 \\
\text { embarazos espontáneos }\end{array}$ & $\begin{array}{l}\text { Los embarazos mediante FIV se asociaron con } \\
\text { prematuridad (RM }=2.36, I C 95 \%=1.28-4.37) \text { y bajo } \\
\text { peso al nacimiento }(\mathrm{RM}=1.89, \mathrm{IC} 95 \%=1.03-3.46)\end{array}$ & 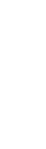 \\
\hline $\begin{array}{l}\text { Farhi et al., } \\
2013^{21}\end{array}$ & $\begin{array}{l}\text { Israel, } \\
\text { 1997-2004 }\end{array}$ & $\begin{array}{l}\text { Cohorte } \\
\text { retrospectiva }\end{array}$ & $\begin{array}{l}\text { Cohorte expuesta: } \\
9042 \text { FIV-ICSI } \\
\text { Cohorte no expuesta: } \\
213288 \text { embarazos } \\
\text { espontáneos }\end{array}$ & 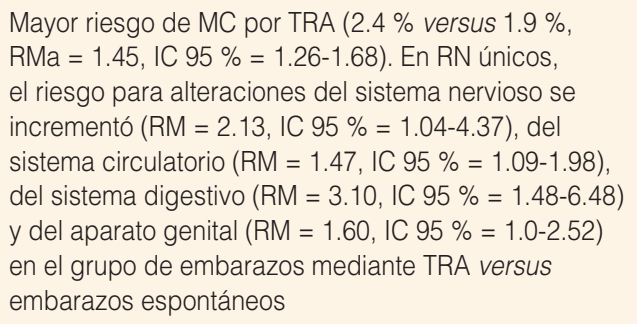 & 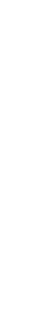 \\
\hline $\begin{array}{l}\text { Harlev et al., } \\
2018^{15}\end{array}$ & $\begin{array}{l}\text { Israel, } \\
\text { enero 1991- } \\
\text { diciembre } 2013\end{array}$ & $\begin{array}{l}\text { Casos y } \\
\text { controles }\end{array}$ & $\begin{array}{l}\text { Casos: } 229 \text { FIV } \\
\text { Controles: } 7929 \text { embarazos } \\
\text { espontáneos }\end{array}$ & $\begin{array}{l}\text { Parto prematuro }(p<0.001) \text {, restricción del } \\
\text { crecimiento }(p<0.001) \text { y cesárea }(p<0.001) \text { tuvieron } \\
\text { alta prevalencia en embarazos por TRA (FIV) }\end{array}$ & 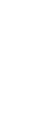 \\
\hline $\begin{array}{l}\text { Healy et al., } \\
2010^{42}\end{array}$ & $\begin{array}{l}\text { Australia, } \\
\text { 1991-2004 }\end{array}$ & $\begin{array}{l}\text { Cohorte } \\
\text { retrospectiva }\end{array}$ & $\begin{array}{l}\text { Cohorte expuesta: } \\
\text { 6730 FIV-ICSI. } \\
\text { Cohorte no expuesta: } 24619 \\
\text { embarazos espontáneos }\end{array}$ & $\begin{array}{l}\text { El grupo de FIV-ICSI presentó mayor hemorragia } \\
\text { posparto }(6.7 \% \text { versus } 3.6 \%, \mathrm{RMa}=2.0 \\
\text { IC } 95 \%=1.8-2.3)\end{array}$ & $\frac{1}{0}$ \\
\hline $\begin{array}{l}\text { Henningsen } \\
\text { et al., } 2011^{16}\end{array}$ & $\begin{array}{l}\text { Dinamarca, } \\
\text { 1994-2008 }\end{array}$ & $\begin{array}{l}\text { Cohorte } \\
\text { retrospectiva }\end{array}$ & $\begin{array}{l}\text { Cohorte expuesta: } 3881 \\
\text { FIV-ICSI } \\
\text { Cohorte no expuesta: } 7758 \\
\text { embarazos espontáneos }\end{array}$ & $\begin{array}{l}\text { Mayor riesgo de BPN (RM = 1.4, IC } 95 \%=1.1-1.7) \text { y } \\
\text { prematuridad (RM = 1.3, IC } 95 \%=1.1-1.6) \text { con FIV- } \\
\text { ICSI en comparación con embarazos espontáneos }\end{array}$ & 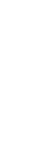 \\
\hline $\begin{array}{l}\text { Jackson et al., } \\
2015^{38}\end{array}$ & $\begin{array}{l}\text { Estados Unidos, } \\
\text { enero } \\
2000 \text {-octubre } \\
2010\end{array}$ & $\begin{array}{l}\text { Cohorte } \\
\text { retrospectiva }\end{array}$ & $\begin{array}{l}\text { Cohorte expuesta: } 185 \text { FIV } \\
\text { Cohorte no expuesta: } 193 \\
\text { embarazos espontáneos }\end{array}$ & $\begin{array}{l}\text { Aumento en el riesgo de acretismo placentario ( } 2.7 \% \\
\text { versus } 0 \% \text { ) y de cesárea electiva ( } 75.1 \% \text { versus } \\
49.7 \% \text { ) en mujeres que concibieron mediante TRA } \\
\text { comparadas con aquellas con embarazo espontáneo }\end{array}$ & 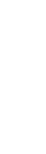 \\
\hline $\begin{array}{l}\text { Källen et al., } \\
2010^{6}\end{array}$ & $\begin{array}{l}\text { Suecia, } \\
\text { 1982-2007 }\end{array}$ & $\begin{array}{l}\text { Cohorte } \\
\text { retrospectiva }\end{array}$ & $\begin{array}{l}\text { Cohorte expuesta: } 1545 \\
\text { gemelos por FIV } \\
\text { Cohorte no expuesta: } 8675 \\
\text { gemelos espontáneos }\end{array}$ & $\begin{array}{l}\text { Mayor riesgo de nacimiento prematuro < } 32 \text { SDG } \\
(\mathrm{RMa}=1.52, \mathrm{IC} 95 \%=1.18-1.97), \mathrm{BPN}<500 \mathrm{~g} \\
(\mathrm{RM}=1.54, \mathrm{IC} 95 \%=1.25-1.89) \text { en el grupo de FIV }\end{array}$ & 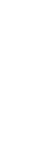 \\
\hline $\begin{array}{l}\text { Källén et al., } \\
2010^{37}\end{array}$ & $\begin{array}{l}\text { Suecia, } \\
\text { 1982-2007 }\end{array}$ & $\begin{array}{l}\text { Casos y } \\
\text { controles }\end{array}$ & $\begin{array}{l}\text { Casos: } 15570 \text { FIV } \\
\text { Controles: } 689157 \\
\text { embarazos espontáneos }\end{array}$ & $\begin{array}{l}\text { Incremento en el riesgo de malformaciones congénitas } \\
(\mathrm{RM}=1.23, \mathrm{IC} 95 \%=1.14-1.32) \text { relacionadas con FIV }\end{array}$ & 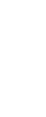 \\
\hline $\begin{array}{l}\text { Kosteria et al., } \\
2017^{39}\end{array}$ & Grecia & $\begin{array}{l}\text { Casos y } \\
\text { controles }\end{array}$ & $\begin{array}{l}\text { Casos: } 42 \text { ICSI. } \\
\text { Control: } 42 \text { embarazos } \\
\text { espontáneos }\end{array}$ & $\begin{array}{l}\text { El grupo ICSI tuvo una gestación más corta, más } \\
\text { cesáreas }(p=0.003) \text {, menor peso y longitud al } \\
\text { nacimiento }\end{array}$ & $\stackrel{\frac{c}{n}}{n}$ \\
\hline
\end{tabular}


Tabla 1. Características de estudios publicados en revistas indexadas entre 2010 y 2018, sobre complicaciones obstétricas y perinatales en embarazos por FIV e ICSI y concepciones espontáneas (Continuación)

\begin{tabular}{|c|c|c|c|c|}
\hline Estudio & País, periodo & Diseño & Población & Complicaciones reportadas \\
\hline $\begin{array}{l}\text { Lerner-Geva } \\
\text { et al, } 2017^{27}\end{array}$ & $\begin{array}{l}\text { Israel, } \\
\text { 1994-2004 }\end{array}$ & $\begin{array}{l}\text { Cohorte } \\
\text { histórica }\end{array}$ & $\begin{array}{l}\text { Cohorte expuesta: } 9042 \text { FIV } \\
\text { Cohorte no expuesta: } \\
211763 \text { espontáneos }\end{array}$ & $\begin{array}{l}\text { Los niños concebidos por TRA tuvieron riesgo } \\
\text { significativo para cáncer específico como } \\
\text { retinoblastoma (RM }=6.18 \text {, IC } 95 \%=1.22-31.2) \text { y } \\
\text { tumores renales }(\mathrm{RM}=3.25, \mathrm{IC} 95 \%=1.67-6.32)\end{array}$ \\
\hline $\begin{array}{l}\text { Messerschmidt } \\
\text { et al., } 2010^{32}\end{array}$ & $\begin{array}{l}\text { Austria, } \\
\text { 1999-2007 }\end{array}$ & $\begin{array}{l}\text { Cohorte } \\
\text { retrospectiva }\end{array}$ & $\begin{array}{l}\text { Cohorte expuesta: } 195 \text { FIV } \\
\text { Cohorte no expuesta: } 1228 \\
\text { embarazos espontáneos }\end{array}$ & $\begin{array}{l}\text { Los nacimientos pretérmino fueron mayores en los } \\
\text { embarazos múltiples obtenidos por FIV }(81.8 \%) \text { a } \\
\text { diferencia de los espontáneos }(57.2 \%) \text { y PEG }(p<0.01)\end{array}$ \\
\hline $\begin{array}{l}\text { Min Yang et al., } \\
2018^{31}\end{array}$ & $\begin{array}{l}\text { China, } \\
\text { 2006-2016 }\end{array}$ & $\begin{array}{l}\text { Cohorte } \\
\text { retrospectiva }\end{array}$ & $\begin{array}{l}\text { Cohorte expuesta: } 2484 \text { FIV } \\
\text { Cohorte no expuesta: } \\
109559 \text { embarazos } \\
\text { espontáneos }\end{array}$ & $\begin{array}{l}\text { Las concepciones por TRA tuvieron mayor riesgo para } \\
\text { cualquier tipo defecto al nacimiento comparadas con } \\
\text { los embarazos espontáneos } \\
\text { (RMa 2.10, IC } 95 \%=1.63-2.69 \text { ) }\end{array}$ \\
\hline $\begin{array}{l}\text { Moini et al., } \\
2012^{18}\end{array}$ & $\begin{array}{l}\text { Irán, } \\
\text { enero } \\
2008 \text {-octubre } \\
2010\end{array}$ & $\begin{array}{l}\text { Cohorte } \\
\text { prospectiva }\end{array}$ & $\begin{array}{l}\text { Cohorte expuesta: } 420 \text { FIV } \\
\text { Cohorte no expuesta: } 340 \\
\text { embarazos espontáneos }\end{array}$ & $\begin{array}{l}\text { Las tasas de nacimiento prematuro }(\mathrm{RM}=5.2 \text {, } \\
\text { IC } 95 \%=2.1-12.9), \mathrm{BPN}(\mathrm{RM}=2.2, \mathrm{IC} 95 \%=1.0- \\
\text { 3.9), ingreso a UCIN (RM = } 2.0, \mathrm{IC} 95 \%=1.2-3.2) \\
\text { y mortalidad perinatal (RM }=2.3, \mathrm{IC} 95 \%=1.2-4.0) \\
\text { fueron significativos en TRA }\end{array}$ \\
\hline $\begin{array}{l}\text { Mozafari } \\
\text { Kermani et al., } \\
2018^{36}\end{array}$ & $\begin{array}{l}\text { Irán, } \\
\text { enero } \\
2012 \text {-diciembre } \\
2014\end{array}$ & $\begin{array}{l}\text { Cohorte } \\
\text { histórica }\end{array}$ & $\begin{array}{l}\text { Cohorte expuesta: } 168 \\
\text { FIV-ICSI. Cohorte no } \\
\text { expuesta: } 652 \text { embarazos } \\
\text { espontáneos }\end{array}$ & $\begin{array}{l}\text { Los niños concebidos por TRA tuvieron dos veces } \\
\text { mayor riesgo de presentar MC ( } p=0.046) \text { en } \\
\text { comparación con los concebidos mediante embarazo } \\
\text { espontáneo }\end{array}$ \\
\hline $\begin{array}{l}\text { Opdahl et al., } \\
2015^{43}\end{array}$ & $\begin{array}{l}\text { Suecia, } \\
\text { Dinamarca y } \\
\text { Noruega, } \\
\text { 1988-2007 }\end{array}$ & $\begin{array}{l}\text { Cohorte } \\
\text { retrospectiva }\end{array}$ & $\begin{array}{l}\text { Cohorte expuesta: } \\
58006 \text { FIV-ICSI Cohorte } \\
\text { no expuesta: } 315273 \\
\text { embarazos espontáneos }\end{array}$ & $\begin{array}{l}\text { El riesgo de trastornos hipertensivos del embarazo fue } \\
\text { mayor en el grupo de TRA (RM = } 1.16 \\
\text { IC } 95 \%=1.10-1.21)\end{array}$ \\
\hline $\begin{array}{l}\text { Panagiotopoulou } \\
\text { et al., } 2016^{34}\end{array}$ & $\begin{array}{l}\text { Grecia, } \\
\text { 1995-2012 }\end{array}$ & $\begin{array}{l}\text { Cohorte } \\
\text { retrospectiva }\end{array}$ & $\begin{array}{l}\text { Cohorte expuesta: } 389 \\
\text { FIV-ICSI. } \\
\text { Cohorte no expuesta: } 485 \\
\text { embarazos espontáneos }\end{array}$ & $\begin{array}{l}\text { En el grupo de TRA, } 8.2 \% \text { presentó enfermedad } \\
\text { congénita cardiovascular en comparación con } 4.3 \% \\
\text { de los concebidos naturalmente }(\mathrm{RM}=1.90 \\
\text { IC } 95 \%=1.08-3.34, p=0.024)\end{array}$ \\
\hline $\begin{array}{l}\text { Pelkonen et al., } \\
2014^{11}\end{array}$ & $\begin{array}{l}\text { Finlandia, } \\
\text { 1995-2006 }\end{array}$ & $\begin{array}{l}\text { Cohorte } \\
\text { retrospectiva }\end{array}$ & $\begin{array}{l}\text { Cohorte expuesta: } 4772 \\
\text { FIV. Cohorte no expuesta: } \\
31243 \text { embarazos } \\
\text { espontáneos }\end{array}$ & $\begin{array}{l}\text { Los niños de embarazos por TRA tuvieron más riesgo } \\
\text { de } \mathrm{MC} \text { mayor (RM =1.24, IC } 95 \%=1.05-1.47) \text { en } \\
\text { comparación con los concebidos espontáneamente }\end{array}$ \\
\hline $\begin{array}{l}\text { Poon et al., } \\
2013^{22}\end{array}$ & $\begin{array}{l}\text { Singapur, } \\
\text { noviembre 2001- } \\
\text { enero } 2012\end{array}$ & $\begin{array}{l}\text { Cohorte } \\
\text { retrospectiva }\end{array}$ & $\begin{array}{l}\text { Cohorte expuesta: } 536 \\
\text { FIV-ICSI } \\
\text { Cohorte no expuesta: } \\
16335 \text { espontáneos }\end{array}$ & $\begin{array}{l}\text { Los embarazos por TRA se asociaron a mayor riesgo } \\
\text { de prematuridad }(\mathrm{RM}=5.95 \\
\text { IC } 95 \%=4.99-7.08) \text {, BPN }(\mathrm{RM}=5.54 \\
\text { IC } 95 \%=4.37-6.13) \text { y mortalidad perinatal } \\
\text { (RM }=4.33, \text { IC } 95 \%=2.05-9.12)\end{array}$ \\
\hline $\begin{array}{l}\text { Raatikainen } \\
\text { et al., } 2012^{19}\end{array}$ & $\begin{array}{l}\text { Finlandia, } \\
\text { 1989-2007 }\end{array}$ & $\begin{array}{l}\text { Casos y } \\
\text { controles }\end{array}$ & $\begin{array}{l}\text { Casos: } 428 \text { FIV/ICSI. } \\
\text { Controles: } 18984 \\
\text { embarazos espontáneos }\end{array}$ & $\begin{array}{l}\text { Las TRA aumentaron significativamente los riesgos de } \\
\text { parto prematuro (RM }=2.72, \text { IC } 95 \%=1.02-7.22) \text { y } \\
\text { BPN }(\mathrm{RM}=1.92, \operatorname{IC} 95 \%=1.31-2.81)\end{array}$ \\
\hline $\begin{array}{l}\text { Sazonova et al., } \\
2011^{17}\end{array}$ & $\begin{array}{l}\text { Suecia, } \\
\text { 2002-2006 }\end{array}$ & $\begin{array}{l}\text { Cohorte } \\
\text { retrospectiva }\end{array}$ & $\begin{array}{l}\text { Cohorte expuesta: } 13544 \\
\text { FIV } \\
\text { Cohorte no expuesta: } \\
587009 \text { sin TRA }\end{array}$ & $\begin{array}{l}\text { La FIV se relacionó con mayor riesgo de prematuridad } \\
<28 \mathrm{SDG}(\mathrm{RMa}=1.69, \mathrm{IC} 95 \%=1.34-2.14), \mathrm{BPN} \\
<2500 \mathrm{~g}(\mathrm{RMa}=1.91, \mathrm{IC} 95 \%=1.79-2.04) \mathrm{y} \\
\text { peso }<1500 \mathrm{~g}(\mathrm{RMa}=1.72, \mathrm{IC} 95 \%=1.49-1.99) \\
\text { comparada con embarazo espontáneo }\end{array}$ \\
\hline $\begin{array}{l}\text { Sazonova et al., } \\
2012^{40}\end{array}$ & $\begin{array}{l}\text { Suecia, } \\
\text { 2002-2006 }\end{array}$ & $\begin{array}{l}\text { Cohorte } \\
\text { retrospectiva }\end{array}$ & $\begin{array}{l}\text { Cohorte expuesta: } 11292 \\
\text { FIV Cohorte no expuesta: } \\
571914 \text { embarazos } \\
\text { espontáneos }\end{array}$ & $\begin{array}{l}\text { Las TRA estuvieron relacionados con mayor tasa de } \\
\text { prematuridad (RMa }=1.92 \text {, IC } 95 \%=1.12-3.93) \text {, } \\
\text { macrosomía ( } \mathrm{RMa}=1.29, \mathrm{IC} 95 \%=1.04-1.59) \text { y } \\
\text { preeclampsia ( } \mathrm{RMa}=1.25, \mathrm{IC} 95 \%=1.03-1.51 \text { ) } \\
\text { comparadas con embarazos espontáneos }\end{array}$ \\
\hline Sun et al., $201^{48}$ & $\begin{array}{l}\text { China, } \\
\text { 2004-2008 }\end{array}$ & $\begin{array}{l}\text { Casos y } \\
\text { controles }\end{array}$ & $\begin{array}{l}\text { Casos: } 1327 \text { FIV/ICSI } \\
\text { Control: } 5222 \text { embarazos } \\
\text { espontáneos }\end{array}$ & $\begin{array}{l}\text { El grupo con TRA tuvo mayor incidencia de cesárea } \\
(\mathrm{RM}=1.40 ; \text { IC } 95 \%=1.24-1.60) \text {, prematuridad y bajo } \\
\text { peso al nacimiento }(p<0.001)\end{array}$ \\
\hline
\end{tabular}


Tabla 1. Características de estudios publicados en revistas indexadas entre 2010 y 2018, sobre complicaciones obstétricas y perinatales en embarazos por FIV e ICSI y concepciones espontáneas (Continuación)

\begin{tabular}{|c|c|c|c|c|}
\hline Estudio & País, periodo & Diseño & Población & Complicaciones reportadas \\
\hline $\begin{array}{l}\text { Sundh et al., } \\
2014^{59}\end{array}$ & $\begin{array}{l}\text { Suecia, } \\
\text { Dinamarca, } \\
\text { Finlandia y } \\
\text { Noruega, } \\
\text { 1982-2007 }\end{array}$ & $\begin{array}{l}\text { Cohorte } \\
\text { retrospectiva }\end{array}$ & $\begin{array}{l}\text { Cohorte expuesta: } \\
91796 \text { FIV-ICSI Cohorte } \\
\text { no expuesta: } 358419 \\
\text { embarazos espontáneos }\end{array}$ & $\begin{array}{l}\text { Se observó riesgo aumentado para dos de } 12 \text { tipos } \\
\text { de cáncer en el grupo de TRA: tumores del SNC, } \\
\text { RMa }=1.44 \text { e IC } 95 \%=1.01-2.05 \text { y neoplasias } \\
\text { epiteliales malignas, RMa }=2.03 \text { e IC } 95 \%=1.06-3.89\end{array}$ \\
\hline $\begin{array}{l}\text { Suzuki et al., } \\
2010^{25}\end{array}$ & $\begin{array}{l}\text { Japón, } \\
\text { 2000-2007 }\end{array}$ & $\begin{array}{l}\text { Casos y } \\
\text { controles }\end{array}$ & $\begin{array}{l}\text { Casos: } 64 \text { FIV } \\
\text { Controles: } 87 \text { embarazos } \\
\text { espontáneos. }\end{array}$ & $\begin{array}{l}\text { La tasa de cesáreas en el grupo de FIV fue } \\
\text { mayor }(86 \%) \text { que en en el grupo control }(67 \%) \text { y } \\
\text { estadísticamente significativa }(p<0.01)\end{array}$ \\
\hline $\begin{array}{l}\text { Tomic et al., } \\
2011^{60}\end{array}$ & $\begin{array}{l}\text { Croacia, } \\
2006-2009\end{array}$ & $\begin{array}{l}\text { Casos y } \\
\text { controles }\end{array}$ & $\begin{array}{l}\text { Casos: } 283 \text { FIV. Controles: } \\
283 \text { espontáneos. }\end{array}$ & $\begin{array}{l}\text { Los embarazos únicos con FIV en primíparas } \geq 35 \text { años } \\
\text { tuvieron mayor número de cesáreas en comparación } \\
\text { con los espontáneos }(p<0.0001) \text { y BPN }(p<0.05)\end{array}$ \\
\hline $\begin{array}{l}\text { Toshimitsu et al., } \\
2014^{26}\end{array}$ & $\begin{array}{l}\text { Japón, } \\
\text { 2006-2010 }\end{array}$ & $\begin{array}{l}\text { Casos y } \\
\text { controles }\end{array}$ & $\begin{array}{l}\text { Casos: } 88 \text { FIV-ICSI } \\
\text { Control: } 242 \text { embarazos } \\
\text { espontáneos }\end{array}$ & $\begin{array}{l}\text { Aumento estadísticamente significativo en hipertensión } \\
\text { gestacional en TRA ( } p 0.002)\end{array}$ \\
\hline $\begin{array}{l}\text { Valenzuela- } \\
\text { Alcaraz et al., } \\
2018^{33}\end{array}$ & $\begin{array}{l}\text { España, } \\
\text { 2014-2016 }\end{array}$ & $\begin{array}{l}\text { Cohorte } \\
\text { prospectiva }\end{array}$ & $\begin{array}{l}\text { Cohorte expuesta: } 50 \text { FIV- } \\
\text { ICSICohorte no expuesta: } \\
50 \text { espontáneos }\end{array}$ & $\begin{array}{l}\text { Los fetos gemelos de TRA mostraron cambios } \\
\text { cardiovasculares significativos con predominio de } \\
\text { afección del corazón derecho }(p<0.001)\end{array}$ \\
\hline $\begin{array}{l}\text { Wen et al., } \\
2010^{28}\end{array}$ & $\begin{array}{l}\text { Canadá, } \\
\text { 1996-2005 }\end{array}$ & $\begin{array}{l}\text { Cohorte } \\
\text { retrospectiva }\end{array}$ & $\begin{array}{l}\text { Cohorte expuesta: } 1044 \\
\text { FIV-ICSI } \\
\text { Cohorte no expuesta: } 1910 \\
\text { embarazos espontáneos }\end{array}$ & $\begin{array}{l}\text { Las TRA estuvieron relacionadas con mayor riesgo } \\
\text { de preeclampsia }(\mathrm{RMa}=2.15, \mathrm{IC} 95 \%=1.33-3.46) \text { y } \\
\text { mayor cantidad de } \mathrm{MC} \text { cardiacas en comparación con } \\
\text { los embarazos espontáneos }(\mathrm{RMa}=4.58 \\
\text { IC } 95 \%=1.48-14.18)\end{array}$ \\
\hline $\begin{array}{l}\text { Wennberg et al., } \\
201612\end{array}$ & $\begin{array}{l}\text { Suecia, } \\
\text { Dinamarca, } \\
\text { Finlandia y } \\
\text { Noruega, } \\
\text { 1982-2007 }\end{array}$ & $\begin{array}{l}\text { Cohorte } \\
\text { retrospectiva }\end{array}$ & $\begin{array}{l}\text { Cohorte expuesta: } \\
39890 \text { FIV-ICSI Cohorte } \\
\text { no expuesta: } 245600 \\
\text { embarazos espontáneos }\end{array}$ & $\begin{array}{l}\text { El riesgo de prematuridad, BPN, MC, PEG, muerte } \\
\text { fetal, cesárea y hemorragias del embarazo fue } \\
\text { mayor en el grupo de TRA que en el de embarazos } \\
\text { espontáneos }(p<0.001)\end{array}$ \\
\hline $\begin{array}{l}\text { Wisborg et al., } \\
2010^{7}\end{array}$ & $\begin{array}{l}\text { Dinamarca, } \\
1989-2006\end{array}$ & $\begin{array}{l}\text { Cohorte } \\
\text { prospectiva }\end{array}$ & $\begin{array}{l}\text { Cohorte expuesta: } 742 \\
\text { FIV-ICSI } \\
\text { Cohorte no expuesta: } \\
18473 \text { embarazos } \\
\text { espontáneos }\end{array}$ & $\begin{array}{l}\text { Aumento estadísticamente significativo de parto } \\
\text { prematuro }<37 \text { SDG }(\mathrm{RM}=1.53, \mathrm{IC} 95 \%=1.15-2.04) \\
\text { y }<32 \mathrm{SDG}(\mathrm{RM}=2.33, \mathrm{IC} 95 \%=1.17-4.65) \text { en FIV- } \\
\text { ICSI comparado con mujeres fértiles }\end{array}$ \\
\hline $\begin{array}{l}\text { Wisborg et al., } \\
2010^{30}\end{array}$ & $\begin{array}{l}\text { Dinamarca, } \\
\text { 1989-2006 }\end{array}$ & $\begin{array}{l}\text { Cohorte } \\
\text { prospectiva }\end{array}$ & $\begin{array}{l}\text { Cohorte expuesta: } 730 \\
\text { FIV-ICSI } \\
\text { Cohorte no expuesta: } \\
18545 \text { embarazos } \\
\text { espontáneos }\end{array}$ & $\begin{array}{l}\text { Embarazos por FIV-ICSI se relacionaron con mayor } \\
\text { riesgo de muerte fetal }(\mathrm{RMa}=4.08, \mathrm{IC} 95 \%=2.11-7.93)\end{array}$ \\
\hline $\begin{array}{l}\text { Yang et al., } \\
2014^{10}\end{array}$ & $\begin{array}{l}\text { China, } \\
\text { enero-diciembre } \\
2011\end{array}$ & $\begin{array}{l}\text { Casos y } \\
\text { controles }\end{array}$ & $\begin{array}{l}\text { Casos: } 1139 \text { FIV-ICSI } \\
\text { Controles: } 111264 \\
\text { embarazos espontáneos }\end{array}$ & $\begin{array}{l}\text { El grupo de embarazos por FIV-ICSI presentó mayor } \\
\text { incidencia de HTA }(\mathrm{RM}=1.27 \text {. IC } 95 \%=1.04-1.60) \text {, } \\
\text { parto prematuro }(\mathrm{RM}=4.53, \mathrm{IC} 95 \%=3.91-5.25) \text {, } \\
\text { diabetes gestacional }(\mathrm{RM}=3.05, \mathrm{IC} 95 \%=2.57-3.60) \\
\text { y placenta previa }(\mathrm{RM}=2.18, \mathrm{IC} 95 \%=1.62-2.94)\end{array}$ \\
\hline $\begin{array}{l}\text { Yu HT et al., } \\
2018^{13}\end{array}$ & $\begin{array}{l}\text { China, } \\
\text { 2005-2016 }\end{array}$ & $\begin{array}{l}\text { Cohorte } \\
\text { retrospectiva }\end{array}$ & $\begin{array}{l}\text { Cohorte expuesta: } 6372 \\
\text { FIV-ICSI } \\
\text { Cohorte no expuesta: } \\
2182179 \text { embarazos } \\
\text { espontáneos }\end{array}$ & $\begin{array}{l}\text { Alto riesgo de prematuridad ( } p=0.025) \text {, BPN }<2500 \mathrm{~g} \\
y<1500 \mathrm{~g}(p<0.001) \text { en el grupo de TRA }\end{array}$ \\
\hline Zhu et al., $2016^{24}$ & $\begin{array}{l}\text { China, } \\
\text { 2006-2014 }\end{array}$ & $\begin{array}{l}\text { Cohorte } \\
\text { retrospectiva }\end{array}$ & $\begin{array}{l}\text { Cohorte expuesta: } 2641 \\
\text { FIV-ICSI } \\
\text { Cohorte no expuesta: } 5282 \\
\text { embarazos espontáneos }\end{array}$ & $\begin{array}{l}\text { Los embarazos por TRA se asociaron a mayor riesgo } \\
\text { de diabetes gestacional (RM }=1.99, I C 95 \%=1.69- \\
2.36) \text {, hipertensión gestacional }(\mathrm{RMa}=2.58, \mathrm{IC} 95 \% \\
=2.11-3.15) \text {, placenta previa (RM }=2.23, \mathrm{IC} 95 \%= \\
1.79-2.78) \text {, hemorragia posparto ( } \mathrm{RM}=2.72, \mathrm{IC} 95 \% \\
=2.18-3.41 \text { ) y adherencias placentarias }(\mathrm{RM}=2.37, \mathrm{IC} \\
95 \%=1.90-2.95) \text {. }\end{array}$ \\
\hline
\end{tabular}

BPN = bajo peso al nacimiento, RPM = ruptura prematura de membrana, DM = diabetes mellitus, HTA = hipertensión arterial, IC = intervalo de confianza, ICSI = inyección intracitoplasmática de espermatozoides, IMC = índice de masa corporal, FIV = fertilización in vitro, MC = malformaciones congénitas, RM = razón de momios, RMa = razón de momios ajustada, $\mathrm{PEG}=$ pequeño para la edad gestacional, $\mathrm{RN}=$ recién nacido, $\mathrm{SDG}=$ semanas de gestación, TRA = técnicas de reproducción asistida, $\mathrm{UCIN}=$ unidad de cuidadoS intensivos neonatales, RPM = ruptura prematura de membranas. 
espontáneas, se reportó tendencia incrementada de encontrar algún defecto congénito en los embarazos mediante TRA. La diferencia fue significativa en siete estudios. ${ }^{11,13,21,27,28,31,33-37}$

- Cesárea: Veintitrés artículos, en los que se registraron 47482 concepciones por FIV-ICSI, registraron cesárea electiva o de emergencia, las cuales representaron $52 \%$ de las complicaciones. En 14 estudios, la indicación de cesárea fue significativa en embarazos mediante TRA al compararlos con embarazos espontáneos. . $-10,12,15,19,22,23,25,33,34,38-40^{-10}$

- Ruptura prematura de membranas (RPM) y pretérmino: la RPM es definida como la ruptura de membranas antes del inicio del trabajo de parto; se denomina pretérmino cuando sucede antes de las 37 semanas de gestación. ${ }^{41}$ Nueve artículos ( $24 \%$ del total) mencionaron RPM y RPM pretérmino (1322 concepciones por TRA), tres indicaron resultados significativos en comparación con embarazos espontáneos. . $^{17,23,24}$

- Hemorragia del embarazo: se consideró hemorragia del embarazo al sangrado debido a placenta previa o desprendimiento prematuro de placenta, complicación registrada en 13 artículos (35\% del total), que incluyeron 2110 embarazos por FIV-ICSI; en cinco estudios los resultados fueron significativos al compararlos con los de embarazos espontáneos. ${ }^{9,12,22,24,42}$

- Diabetes gestacional: fue reportada en 17 artículos (46\% del total), que incluyeron 1455 embarazos por FIV-ICSI; cinco reportaron resultados significativos en comparación con los embarazos espontáneos. ${ }^{9,10,22,24,31}$

- Trastornos hipertensivos del embarazo: como tales se consideró a la hipertensión gestacional, la preeclampsia y la eclampsia, registradas en 19 estudios ( $51 \%$ del total), que incluyeron 8416 FIV-ICSI; constituyeron una de las complicaciones más frecuentes. Solo 11 artículos indicaron riesgo significativo en comparación con los embarazos espontáneos. ${ }^{9} 10,12,22,24,26,28,31,33,40,43$

\section{Discusión}

Se ha señalado que las complicaciones en gestaciones múltiples por FIV son similares a las que se presentan en las concepciones espontáneas, no obstante, los embarazos únicos por FIV se asocian a mayor incidencia de complicaciones. ${ }^{44,45}$ Las razones precisas de este incremento no son claras, pero entre los factores potenciales se incluyen características maternas y paternas, condiciones médicas subyacentes asociadas a la subfertilidad e infertilidad, factores del esperma, uso de medicamentos para la fertilidad, condiciones del laboratorio durante el cultivo de embriones, medio de cultivo, criopreservación y descongelación, diagnóstico genético prenatal (si se realiza), diferencias en el manejo obstétrico o una combinación de los anteriores factores. ${ }^{46}$

En la investigación que se presenta, se analizaron 26 artículos de cohortes y 11 de casos y controles, que incluyeron 10717574 nacimientos; 351217 embarazos por TRA y 10366357 embarazos espontáneos.

Las complicaciones perinatales más frecuentes fueron prematuridad y bajo peso al nacimiento. Se ha mencionado que el mayor riesgo para nacimiento pretérmino son las gestaciones múltiples, originadas en mayor proporción en embarazos por TRA. Además, se ha identificado mayor incidencia de partos prematuros en recién nacidos únicos por TRA comparados con niños concebidos por embarazos únicos espontáneos. ${ }^{47}$ En complicaciones maternas se detectó alta proporción de indicación de cesáreas y trastornos hipertensivos del embarazo; las primeras podrían relacionarse con la ansiedad y decisión del médico dadas las condiciones del embarazo. ${ }^{48}$

La cesárea electiva incrementa la morbimortalidad en comparación con el parto vagina ${ }^{49}$ y representa un riesgo de complicación materno-fetal, puesto que se asocia a hemorragia, infecciones, lesiones a órganos adyacentes y tromboembolismo venoso, entre otras consecuencias maternas. ${ }^{50}$ En los neonatos puede presentarse taquipnea transitoria, dificultad respiratoria y afección de la respuesta inmunitaria. ${ }^{51}$

Aun cuando la diabetes gestacional se asocia significativamente con factores como obesidad, ${ }^{52}$ índice de masa corporal $>30,{ }^{53}$ edad $>35$ años, resistencia a la insulina y niveles elevados de triglicéridos, ${ }^{54}$ en cinco artículos se registró que el riesgo de presentarla fue significativamente mayor en embarazos por TRA, en comparación con concepciones espontáneas.

Las malformaciones congénitas cardiovasculares fueron las más comunes, similar a lo reportado en otra investigación. ${ }^{55}$

Una fortaleza de nuestro trabajo fue el periodo estudiado (casi una década), la selección de artículos que consideraran embarazos espontáneos y las diferentes procedencias de los artículos.

Principalmente en poblaciones europeas y de Estados Unidos se ha encontrado que la FIV y la ICSI incrementan las complicaciones descritas, sin 
embargo se requiere más investigación al respecto en Latinoamérica.

En 2012, Pandey et al. ${ }^{44}$ reportaron resultados similares a los del presente trabajo, evidencia de que los efectos adversos persisten a pesar del avance tecnológico en las TRA. En un metaanálisis de embarazos únicos por TRA comparados con espontáneos, Qin et al..$^{56}$ identificaron mayor riesgo de trastornos hipertensivos del embarazo, diabetes gestacional, cesárea, prematuridad y muy bajo peso al nacimiento. Por su parte, Roque et al. ${ }^{57}$ reportaron solo algunas complicaciones maternas (preeclampsia, placenta accreta) al comparar TRA con embriones frescos o descongelados.

Dado que actualmente la guía de práctica clínica Diagnóstico de la pareja infértil y tratamiento con técnicas de baja complejidad ${ }^{58}$ no establece los riesgos que conllevan las TRA, es imprescindible brindar mayor atención y cuidados a los embarazos mediante esos procedimientos.

\section{Conclusiones}

Diferentes factores durante un embarazo espontáneo pueden derivar en complicaciones obstétricas y perinatales, a los que se adicionan otros factores en los embarazos logrados por TRA como FIV o ICSI, ${ }^{59,60}$ los cuales incrementan el riesgo de presentar dichas complicaciones. Las parejas que decidan y sean candidatas a TRA deben recibir información completa y clara que incluya los factores de riesgo y las complicaciones del procedimiento al que se someterán, además de un cuidadoso asesoramiento preconcepcional dirigido a optimizar la salud general, la identificación de desórdenes reproductivos y la posibilidad de que estos embarazos sean manejados como de alto riesgo.

Por último, es necesario llevar a cabo más estudios prospectivos de cohortes para identificar qué factores de la FIV e ICSI influyen en el desarrollo de complicaciones y cómo podrían ser minimizados o evitados.

\section{Bibliografía}

1. International Committee for Monitoring Assisted Reproductive Technology; de Mouzon J, Lancaster P, Nygren KG, Sullivan E, Zegers-Hochschild F, et al. World Collaborative Report on Assisted Reproductive Technology. Hum Reprod. 2009;24:2310-2320.

2. Halliday JL, Ukoumunne OC, Baker HWG, Breheny S, Jaques AM, Garrett $\mathrm{C}$, et al. Increased risk of blastogenesis birth defects, arising in the first 4 weeks of pregnancy, after assisted reproductive technologies. Hum Reprod. 2010;25:59-65.

3. Kaser DJ, Racowsky C. Clinical outcomes following selection of human preimplantation embryos with time-lapse monitoring: a systematic review. Hum Reprod Update. 2014;20:617-631.
4. Ombelet W, Peeraer K, de Sutter P, Gerris J, Bosmans E, Martens G et al. Perinatal outcome of ICSI pregnancies compared with a matched group of natural conception pregnancies in Flanders (Belgium): a cohort study. Reprod Biomed Online. 2005;11:244-253.

5. Berntsen S, Söderström-Anttila V, Wennerholm UB, Laivuori H, Loft A Oldereid NB, et al. The health of children conceived by ART: "the chicken or the egg?" Hum Reprod Update. 2019;25:137-158.

6. Källén B, Finnström $O$, Lindam A, Nilsson E, Nygren KG, Olausson-Otterblad $P$. Selected neonatal outcomes in dizygotic twins after IVF versus non-IVF pregnancies. BJOG. 2010;117:676-682.

7. Wisborg K, Ingerslev HJ, Henriksen TB. In vitro fertilization and preterm delivery, low birth weight, and admission to the neonatal intensive care unit: a prospective follow-up study. Fertil Steril. 2010;94: 2102-2106.

8. Sun LM, Lanes A, Kingdom JCP, Cao H, Kramer M, Wen SW, et al. intrapartum interventions for singleton pregnancies arising from assisted reproductive technologies. J Obstet Gynaecol Can. 2014;36:795-802.

9. Caserta D, Bordi G, Stegagno M, Filippini F, Podagrosi M, Roselli D, et al. Maternal and perinatal outcomes in spontaneous versus assisted conception twin pregnancies. Eur J Obstet Gynecol Reprod Biol. 2014;174:64-69

10. Yang X, Li Y, Li C, Zhang W. Current overview of pregnancy complications and live-birth outcome of assisted reproductive technology in mainland China. Fertil Steril. 2014;101:385-391.

11. Pelkonen S, Hartikainen AL, Ritvanen A, Koivunen R, Martikainen H, Gissler $\mathrm{M}$, et al. Major congenital anomalies in children born after frozen embryo transfer: a cohort study 1995-2006. Hum Reprod. 2014;29:1552-1557.

12. Wennberg AL, Opdahl S, Bergh C, Aaris-Henningsen AK, Gissler M, Romundstad LB, et al. Effect of maternal age on maternal and neonatal outcomes after assisted reproductive technology. Fertil Steril. 2016;106:1142-1149.

13. Yu HT, Yang Q, Sun XX, Chen GW, Qian NS, Cai RZ, et al. Association of birth defects with the mode of assisted reproductive technology in a Chinese data-linkage cohort. Fertil Steril. 2018;109:849-856.

14. Valenzuela-Alcaraz B, Crispi F, Manau D, Cruz-Lemini M, Borras A Balasch J, et al. Differential effect of mode of conception and infertility treatment on fetal growth and prematurity. J Matern Neonatal Med. 2016;29:3879-3884

15. Harlev A, Walfisch A, Oran E, Har-Vardi I, Friger M, Lunenfeld E, et al. The effect of fertility treatment on adverse perinatal outcomes in women aged at least 40 years. Int J Gynecol Obstet. 2018;140:98-104.

16. Henningsen AKA, Pinborg A, Lidegaard Ø, Vestergaard C, Forman ال Andersen AN. Perinatal outcome of singleton siblings born after assisted reproductive technology and spontaneous conception: Danish national sibling-cohort study. Fertil Steril. 2011;95:959-963.

17. Sazonova A, Källen K, Thurin-Kjellberg A, Wennerholm UB, Bergh C. Obstetric outcome after in vitro fertilization with single or double embryo transfer. Hum Reprod. 2011;26:442-450.

18. Moini A, Shiva M, Arabipoor A, Hosseini R, Chehrazi M, Sadeghi M. Obstetric and neonatal outcomes of twin pregnancies conceived by assisted reproductive technology compared with twin pregnancies conceived spontaneously: a prospective follow-up study. Eur J Obstet Gynecol Reprod Biol. 2012:165:29-32.

19. Raatikainen K, Kuivasaari-Pirinen P, Hippeläinen M, Heinonen S. Comparison of the pregnancy outcomes of subfertile women after infertility treatment and in naturally conceived pregnancies. Hum Reprod. 2012:27:1162-1169.

20. Farhi A, Reichman B, Boyko V, Hourvitz A, Ron-El R L-GL. Maternal and neonatal health outcomes following assisted reproduction. Reprod Biomed Online. 2013;26:454-461.

21. Farhi A, Reichman B, Boyko V, Mashiach S, Hourvitz A, Margalioth EJ, et al. Congenital malformations in infants conceived following assisted reproductive technology in comparison with spontaneously conceived infants. J Matern Neonatal Med. 2013:26:1171-1179.

22. Poon WB, Lian WB. Perinatal outcomes of intrauterine insemination/ clomiphene pregnancies represent an intermediate risk group compared with in vitro fertilisation/intracytoplasmic sperm injection and naturally conceived pregnancies. J Paediatr Child Health. 2013;49:733-740.

23. Bassiouny YA, Bayoumi YA, Gouda HM, Hassan AA. Is intracytoplasmic sperm injection (ICSI) associated with higher incidence of congenital anomalies? A single center prospective controlled study in Egypt. J Matern Neonatal Med. 2014;27(3):279-282

24. Zhu L, Zhang Y, Liu Y, Zhang R, Wu Y, Huang Y, et al. Maternal and live-birth outcomes of pregnancies following assisted reproductive technology: a retrospective cohort study. Sci Rep. 2016;6:1-11.

25. Suzuki S, Miyake H. Perinatal outcomes of elderly primiparous dichorionic twin pregnancies conceived by in vitro fertilization compared with those conceived spontaneously. Arch Gynecol Obstet. 2010;281:87-90.

26. Toshimitsu M, Nagamatsu T, Nagasaka T, Iwasawa-Kawai Y, Komatsu A, Yamashita $\mathrm{T}$, et al. Increased risk of pregnancy-induced hypertension and operative delivery after conception induced by in vitro fertilization/ intracytoplasmic sperm injection in women aged 40 years and older. Fertil Steril. 2014;102:1065-1070. 
27. Lerner-Geva L, Boyko V, Ehrlich S, Mashiach S, Hourvitz A, Haas J, et al. Possible risk for cancer among children born following assisted reproductive technology in Israel. Pediatr Blood Cancer. 2017;64:1-6.

28. Wen SW, Leader A, White RR, Léveillé MC, Wilkie V, Zhou J, et al. A comprehensive assessment of outcomes in pregnancies conceived by in vitro fertilization/intracytoplasmic sperm injection. Eur J Obstet Gynecol Reprod Biol. 2010;150:160-165.

29. Vasario E, Borgarello V, Bossotti C, Libanori E, Biolcati M, Arduino S, et al. IVF twins have similar obstetric and neonatal outcome as spontaneously conceived twins: a prospective follow-up study. Reprod Biomed Online. 2010;21:422-428.

30. Wisborg K, Ingerslev HJ, Henriksen TB. IVF and stillbirth: a prospective follow-up study. Hum Reprod. 2010;25:1312-1316.

31. Yang M, Fan XB, Wu JN, Wang JM. Association of assisted reproductive technology and multiple pregnancies with the risks of birth defects and stillbirth: a retrospective cohort study. Sci Rep. 2018:8:1-8.

32. Messerschmidt A, Olischar M, Birnbacher R, Weber M, Pollak A Leitich H. Perinatal outcome of preterm infants < $1500 \mathrm{~g}$ after IVF pregnancies compared with natural conception. Arch Dis Child Fetal Neonatal Ed. 2010:95:225-230

33. Valenzuela-Alcaraz B, Cruz-Lemini M, Rodríguez-López M, Goncé A García-Otero L, Ayuso $\mathrm{H}$, et al. Fetal cardiac remodeling in twin pregnancy conceived by assisted reproductive technology. Ultrasound Obstet Gynecol. 2018;51:94-100

34. Panagiotopoulou O, Fouzas S, Sinopidis X, Mantagos SP, Dimitriou G Karatza AA. Congenital heart disease in twins: the contribution of type of conception and chorionicity. Int J Cardiol. 2016;218:144-149.

35. Boulet SL, Kirby RS, Reefhuis J, Zhang Y, Sunderam S, Cohen B, et al Assisted reproductive technology and birth defects among liveborn infants in Florida, Massachusetts, and Michigan, 2000-2010. JAMA Pediatr. 2016;170:1-9

36. Kermani RM, Farhangniya $M$, Shahzadeh-Fazeli SA, Bagheri $P$ Ashrafi M, Vosough-Taqi-Dizaj A. Congenital malformations in singleton infants conceived by assisted reproductive technologies and singleton infants by natural conception in Tehran, Iran. Int J Fertil Steril. 2018; 11:304-308.

37. Källén B, Finnström O, Lindam A, Nilsson E, Nygren KG, Otterblad PO. Congenital malformations in infants born after in vitro fertilization in Sweden. Birth Defects Res A Clin Mol Teratol. 2010:88:137-143.

38. Jackson S, Hong C, Wang ET, Alexander C, Gregory KD, Pisarska MD. Pregnancy outcomes in very advanced maternal age pregnancies: the impact of assisted reproductive technology. Fertil Steril. 2015;103:76-80.

39. Kosteria I, Tsangaris GT, Gkourogianni A, Anagnostopoulos A, Papadopoulou A, Papassotiriou I, et al. Proteomics of children born after intracytoplasmic sperm injection reveal indices of an adverse cardiometabolic profile. J Endocr Soc. 2017:1:288-301.

40. Sazonova A, Kllen K, Thurin-Kjellberg A, Wennerholm UB, Bergh C. Obstetric outcome in singletons after in vitro fertilization with cryopreserved/thawed embryos. Hum Reprod. 2012;27:1343-1350.

41. Committee on Practice Bulletins-Obstetrics. ACOG practice bulletin no. 188: prelabor rupture of membranes. Obstet Gynecol. 2018;131:e1-e14.

42. Healy DL, Breheny S, Halliday J, Jaques A, Rushford D, Garrett C, et al Prevalence and risk factors for obstetric haemorrhage in 6730 singleton births after assisted reproductive technology in Victoria. Australia. Hum Reprod. 2010;25:265-274.
43. Opdahl S, Henningsen AA, Tiitinen A, Bergh C, Pinborg A, Romundstad PR, et al. Risk of hypertensive disorders in pregnancies following assisted reproductive technology: a cohort study from the CoNARTaS group. Hum Reprod. 2015;30:1724-1731.

44. Pandey S, Shetty A, Hamilton M, Bhattacharya S, Maheshwari A. Obstetric and perinatal outcomes in singleton pregnancies resulting from IVF/ICSI: a systematic review and meta-analysis. Hum Reprod Update. 2012;18:485-503.

45. Schieve LA, Meikle SF, Ferre C, Peterson HB, Jeng G, Wilcox LS. LOW and very low birth weight in infants conceived with use of assisted reproductive technology. N Engl J Med. 2002;346:731-737.

46. Paulson R. Pregnancy outcome after assisted reproductive technology. UpToDate [sitio web]; 2019.

47. Sanchis-Calvo A, Marcos-Puig B, Juan-García L, Morales-Suárez-Varela MM, Abeledo-Gómez A, Balanzá-MacHancosa R, et al. Características de los recién nacidos tras fecundación in vitro. An Pediatr. 2009;70:333-339.

48. Talaulikar VS, Arulkumaran S. Maternal, perinatal and long-term outcomes after assisted reproductive techniques (ART): implications for clinical practice. Eur J Obstet Gynecol Reprod Biol. 2013;170:13-19.

49. Sandall J, Tribe RM, Avery L, Mola G, Visser GH, Homer CS, et al. Short-term and long-term effects of caesarean section on the health of women and children. Lancet. 2018;392:1349-1357.

50. Burke $C$, Allen R. Complications of cesarean birth: clinical recommendations for prevention and management. MCN Am J Matern Child Nurs. 2019;1-7.

51. Blustein J, Liu J. Time to consider the risks of caesarean delivery for long term child health. BMJ. 2015;350:1-4

52. Doherty DA, Magann EF, Francis J, Morrison JC, Newnham JP. Pre-pregnancy body mass index and pregnancy outcomes. Int J Gynecol Obstet. 2006;95:242-247.

53. Torloni MR, Betrán AP, Horta BL, Nakamura MU, Atallah AN, Moron AF, et al. Prepregnancy BMI and the risk of gestational diabetes: A systematic review of the literature with meta-analysis. Obes Rev. 2009;10:194-203.

54. Yen IW, Lee CN, Lin MW, Fan KC, Wei JN, Chen KY, et al Overweight and obesity are associated with clustering of metabolic risk factors in early pregnancy and the risk of GDM. PLoS One. 2019;14:e0225978.

55. Fedder J, Loft A, Parner ET, Rasmussen S, Pinborg A. Neonatal outcome and congenital malformations in children born after ICSI with testicular or epididymal sperm: a controlled national cohort study. Hum Reprod. 2013;28:230-240.

56. Qin JB, Sheng XQ, Wang H, Chen GC, Yang J, Yu H, et al. Worldwide prevalence of adverse pregnancy outcomes associated with in vitro fertilization/intracytoplasmic sperm injection among multiple births: a systematic review and meta-analysis based on cohort studies. Arch Gynecol Obstet. 2017;295:577-597.

57. Roque M, Valle M, Sampaio M, Geber S. Obstetric outcomes after fresh versus frozen-thawed embryo transfers: a systematic review and meta-analysis. JBRA Assist Reprod. 2018;22:253-260.

58. Instituto Mexicano del Seguro Social. Diagnóstico de la pareja infértil y tratamiento con técnicas de baja complejidad. Guía de práctica clínica. México: IMSS; 2012

59. Sundh KJ, Henningsen AKA, Källen K, Bergh C, Romundstad LB, Gissler $\mathrm{M}$, et al. Cancer in children and young adults born after assisted reproductive technology: a Nordic cohort study from the Committee of Nordic ART and Safety (CoNARTaS). Hum Reprod. 2014;29:2050-2057.

60. Tomic V Tomic J. Neonatal outcome of IVF singletons versus naturally conceived in women aged 35 years and over. Arch Gynecol Obstet. 2011;284:1411-1416. 